Editorial

Anaesthesist 2019-68:801-802

https://doi.org/10.1007/s00101-019-00690-9

(c) Springer Medizin Verlag GmbH, ein Teil von Springer Nature 2019

Check for
updates
I. Wolff · B. Wasser

Redaktion Fachzeitschriften Medizin, Springer Medizin Verlag GmbH, Heidelberg, Deutschland

\section{Facharzt-Training Anästhesiologie - das zweite Sonderheft ist da}

\author{
Insgesamt 50 praxisnahe Fallbeispiele \\ kombiniert mit fundiertem \\ Hintergrundwissen
}

Liebe Leser^Innen,

zum DAC 2019 haben wir Ihnen das erste Sonderheft Facharzt-Training Anästhesiologie vorgestellt. Herr Professor Heller, Augsburg, und Herr PD Dr. Breuer, Coburg, die Heftherausgeber, haben für die Vorbereitung auf die Facharztprüfung, gemeinsam mit ausgewählten Expert ${ }^{\star}$ Innen mit 25 Fällen den Beginn einer Fortbildungsreihe geschaffen, die umfassend, aber übersichtlich kompakt und dabei in ganz neuartiger Form klinische Alltagsexpertise und kognitive Wissensgrundlage anhand von konkreten Fallbeispielen vereint.

Zum DIVI-Kongress 2019 können wir Ihnen nun weitere 25 Fallvignetten im gewohnten Konzept vorstellen (• Abb. 1). Der Start in jedes Kapitel ist dabei wieder eine klinische Fragestellung, an die sich dann anschließend der gesamte klinische Wissensinhalt anknüpft. Die Idee hinter dem Konzept ist dem Verständnis geschuldet, dass die Wissensvermittlung ohne Bezug zur Praxis schnell langatmig wird, und dass das Lernen an Fällen sich durch den Anwendungsbezug fester verankert.

Die Resonanzen von Autor ${ }^{\star}$ Innen und Leser ${ }^{\star}$ Innen auf das erste Sonderheft waren so positiv, dass wir überzeugt sind, dass auch Heft 2 großen Anklang finden wird. Die Gesamtthemenauswahl beider Sonderhefte Facharzt-Training Anästhesiologie wurde so gewählt, dass die häufigsten und die anästhesiologisch exklusiven Themen aufbereitet sind. Beide Hefte ergänzen sich, sodass die 50 vorliegenden
Fälle das relevante Wissen für die Facharztprüfung Anästhesiologie abdecken prüfungsgerecht und praxisnah.

Wir danken den Heftherausgebern und Autor*Innen für diese außergewöhnliche, spannende, lehrreiche und dabei unterhaltsame Fortbildungsreihe, die eben nicht nur für die Vorbereitung auf die Facharztprüfung geeignet ist, sondern auch dem einen oder der anderen langjährigen Expert ${ }^{\star}$ In einen neuen Blick eröffnen kann.

Mehr Informationen zum Sonderheft sowie einen Beispielbeitrag finden Sie auf der Website http://bit.ly/SonderheftAnaesthesist (QR-Code zur Seite siehe unten). Als Abonnent ${ }^{\star}$ In und Leser ${ }^{\star}$ In von Der Anaesthesist haben Sie außerdem die Möglichkeit, dieses Sonderheft zum Sonderpreis von $€ 21,00$ bis zum 15.01.2020 zu bestellen. Bitten wenden Sie sich dazu an Marie-Luise Witschel (Marie-Luise. Witschel@springer.com) unter Angabe des folgenden Codes: C0017993.

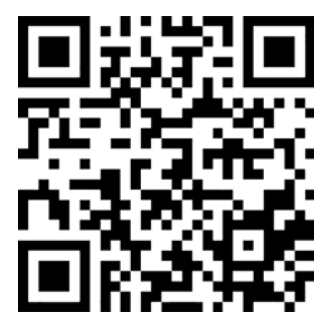

Als krönenden Abschluss zu den beiden Sonderheften werden die 50 Fallvignetten ab Frühjahr 2020 auch als virtuelle Lernkarten auf www.SpringerMedizin. de zum Lernen zur Verfügung stehen, zusammen mit einer kompletten Verlinkung zu vertiefenden Texten und anderen Medien zu allen angesprochenen Themenbereichen. Über den Startpunkt des eLearnings und den Link zum Angebot werden Sie in Der Anaesthesist zuverlässig informiert werden.

Wir wünschen Ihnen viel Freude beim Lernen in dieser regulären Ausgabe von Der Anaesthesist und - wenn Sie wollen - im Sonderheft FacharztTraining Anästhesiologie.

Ihre

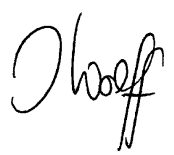

Dr. Ines Wolff (Redaktion Der Anaesthesist) und

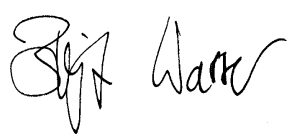

Dr. Birgit Wasser (Verantwortliche Facharzt-Training Anästhesiologie)

\section{Korrespondenzadresse}

Dr. I. Wolff

Redaktion Fachzeitschriften Medizin, Springer Medizin Verlag GmbH

Tiergartenstr. 17, 69121 Heidelberg,

Deutschland

ines.wolff@springer.com

Interessenkonflikt. I. Wolff und B. Wasser geben an, dass kein Interessenkonflikt besteht. 


\section{Editorial}

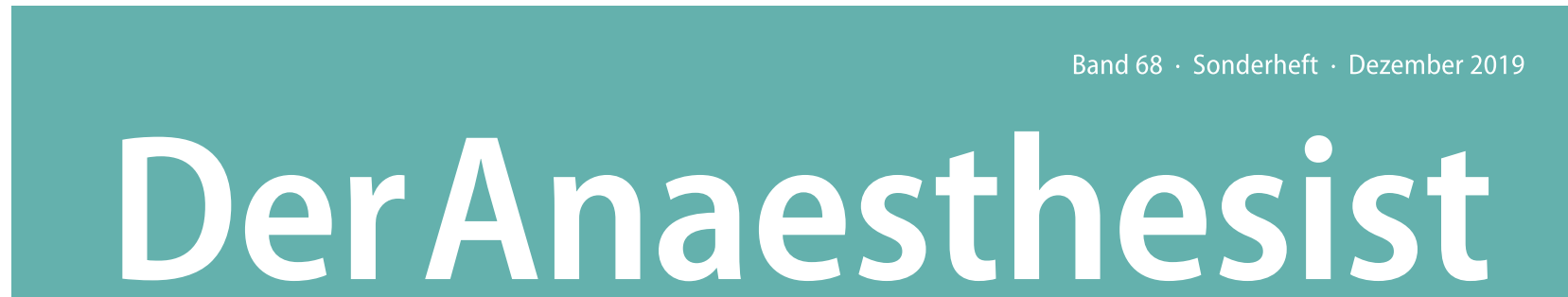

Zeitschrift für Anästhesie • Intensivmedizin .

Notfall- und Katastrophenmedizin · Schmerztherapie

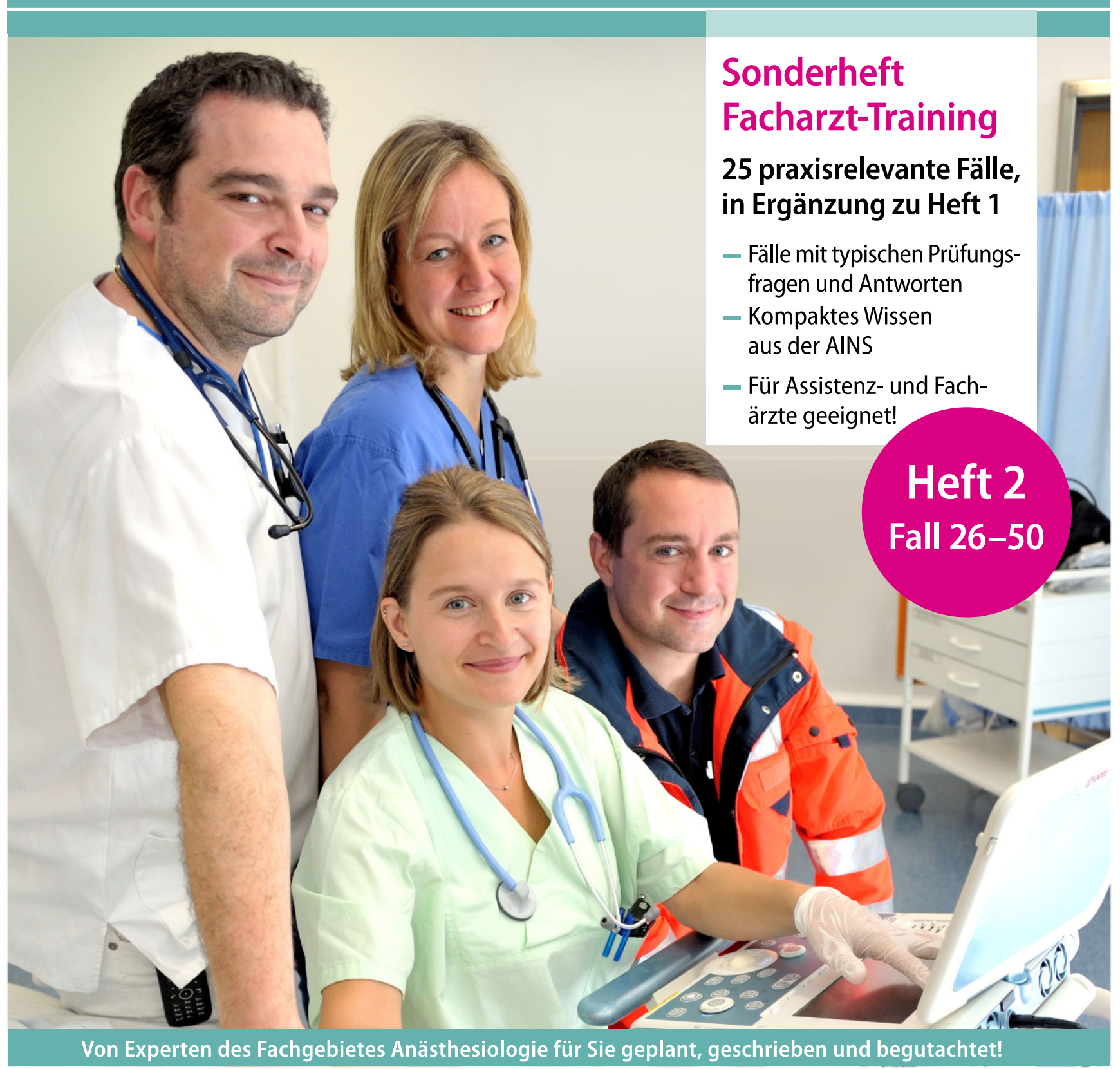

www.springermedizin.de

Springer Medizin

Abb. 1 ॥ Das zweite Sonderheft Facharzt-Training Anästhesiologie ist da! 